\title{
GW23-e1041 CORRELATION OF RENAL RESISTIVE INDEX, TUMOUR NECROSIS $\alpha$ AND INTERLEUKIN 10 WITH HYPERTENSIVE RENAL DAMAGE
}

doi:10.1136/heartjnl-2012-302920a.173

${ }^{1}$ Yang Zhen, ${ }^{2} Y u$ Xin, ${ }^{1}$ Wang Xue-zhong, ${ }^{1}$ Sha Yong, ${ }^{1}$ Wang Jing-jing, ${ }^{1}$ Jia Shao-bin. ${ }^{1}$ Heart Center, the General Hospital of Ningxia Medical University; ${ }^{2}$ School of Laboratory Medicine, Ningxia Medical University

Objectives To investigate the changes of renal resistive index (RRI) and the serum levels of necrosis $\alpha$ (TNF- $\alpha$ ) and interleukin 10 (IL$10)$ in patients with hypertensive renal damage, whereby to explore the correlation of RRI, TNF- $\alpha$ and IL-10 with the hypertensive renal damage.

Methods Seventy three patients with primary hypertension were divided into two groups according to their urinary albumin excretion rate (UAER): normal buminuric hypertensive group $(n=37)$, hypertensive renal damage group $(n=36)$. RRI was measured using Doppler ultrasonography, serum TNF- $\alpha$ and IL-10 using radioimmune assay. Thirty normotensive healthy persons were selected as normotensive control group.

Results RRI and TNF- $\alpha$ were significantly higher and IL-10 significantly lower in patients with essential hypertension than those in normotensive control group $\mathrm{p}<0.5$ ), and in patients with hypertension, those with renal damage had higher RRI and TNF- $\alpha$ and a lower IL-10 than those without $p<0.5$ ), with a statistically significant difference among groups $p<0.5)$. RRI, TNF- $\alpha$ and IL-10 were found to have correlations with UAER $(r=0.801, p<0.01 ; r=0.703$, $\mathrm{p}<0.01 ; \mathrm{r}=-0.613, \mathrm{p}<0.01)$, but no correlation with the level of blood pressure, and RRI positively correlated with TNF- $\alpha$ $(\mathrm{r}=0.609, \mathrm{p}<0.001)$, negatively with IL-10 $(\mathrm{r}=-0.533, \mathrm{p}<0.01)$.

Conclusions RRI is remarkably increased in patients with hypertensive renal damage, whereby can be used as a parameter, together with UAER, in evaluating hypertensive renal damage. TNF- $\alpha$ is increased and IL-10 decreased significantly in patients with hypertensive renal damage, indicating that the imbalanced cytokine network may play a role in the pathological mechanisms of hypertensive renal damage. 